\title{
BILDER-BILDER
}

\section{Koloniale Bildpraktiken und ihre postkolonialen Wiedergänger}

\author{
«Photographs have long played an important mediating \\ role within wider networks of social, political, and \\ economic exchange between Europe and the Pacific». ${ }^{1}$
}

\section{Royale Porträts}

1 Haidy Geismar: Malakula: A Photographic Collection, in: Comparative Studies in Society and History, Bd. 48 , Nr. 3, 2006, 520-563, hier 524 .

2 Vgl. Tessa Wong: Prince Philip: The Vanuatu tribes mourning the death of their (god, in: BBC News, 12.4.2021, bbc.com/news/world-asia56713953 (11.5.2021); Anke Richter: Halbgott mit Schweineknüppel, in: Spiegel Online, 13.4.2021, spiegel. de/panorama/prinz-philip-kult-inder-suedsee-halbgott-mit-schweine knueppel-a-8d6d748a-48cb-43db8722-00071291d4cc (11.5.2021).

3 Vgl. Jill Gralow, Stefica Nicol Bikes, Melanie Burton: Prince Philip devotees hold mourning ceremony in Vanuatu, in: Reuters, 13.4.2021, reuters.com/world/uk/prince-philipdevotees-hold-mourning-ceremonyvanuatu-2021-04-13 (15.6.2021).

4 Zur Mortifizierung durch die Fotografie siehe Roland Barthes: La chambre claire. Note sur la photographie, Paris 1980. Demnach könnte man in den Aufnahmen eine rdoppelte Séance), eine fotografische Geisterbeschwörung des verstorbenen Royal durch die seinen Tod vorwegnehmenden Porträtfotografien sehen.
Als am 9. April 202 I die Nachricht vom Ableben Prinz Philips die Runde machte, dauerte es nicht lange, bis Berichte von der weltweiten Trauer um den britischen Royal über verschiedene Nachrichtenkanäle und Social-Media-Portale verbreitet wurden. Mehrere Sender berichteten darüber, dass der verstorbene Ehepartner der Queen offenbar auch am pazifischen Rand des ehemaligen britischen Empires betrauert wurde. Diese Berichte wurden vielfach in sozialen Medien geteilt und kommentiert und erhielten dadurch erhöhte Aufmerksamkeit. Interesse weckten vor allem Spekulationen über die Ursache der bislang nur wenig bekannten Nähe zwischen dem britischen Königshaus und Menschen im Südpazifik. BBC News und Spiegel Online zeigten sich verwundert über die intensive Anteilnahme, die insbesondere die männliche Bevölkerung von Yakel und Yaohnanen, zwei Siedlungen auf der Insel Tanna im Inselstaat Vanuatu, an Philips Tod zeigte. ${ }^{2}$ Presseberichten zufolge kamen Menschen dort zusammen, um gemeinsam zu trauern. ${ }^{3}$ Publik gemachte Aufnahmen zeigen Männer, die Fotografien des Prinzen (und des britischen Königspaars) in die Kamera halten (Abb. I).

Mehr als die Nachricht selbst sind es diese Fotografien von Fotografien, die mich ansprechen - nicht nur, weil sie den Tod eines Menschen anzeigen, der bereits durch frühere fotografische Aufnahmen mortifiziert worden ist, ${ }^{4}$ sondern weil sie vielmehr ein spezifisches Handeln mit Fotografien bezeugen, das verschiedene Orte und Zeiten miteinander verbindet. Es sind so gesehen Bilder-Bilder, in denen zwei verschiedene Zeitschichten und konkurrierende 


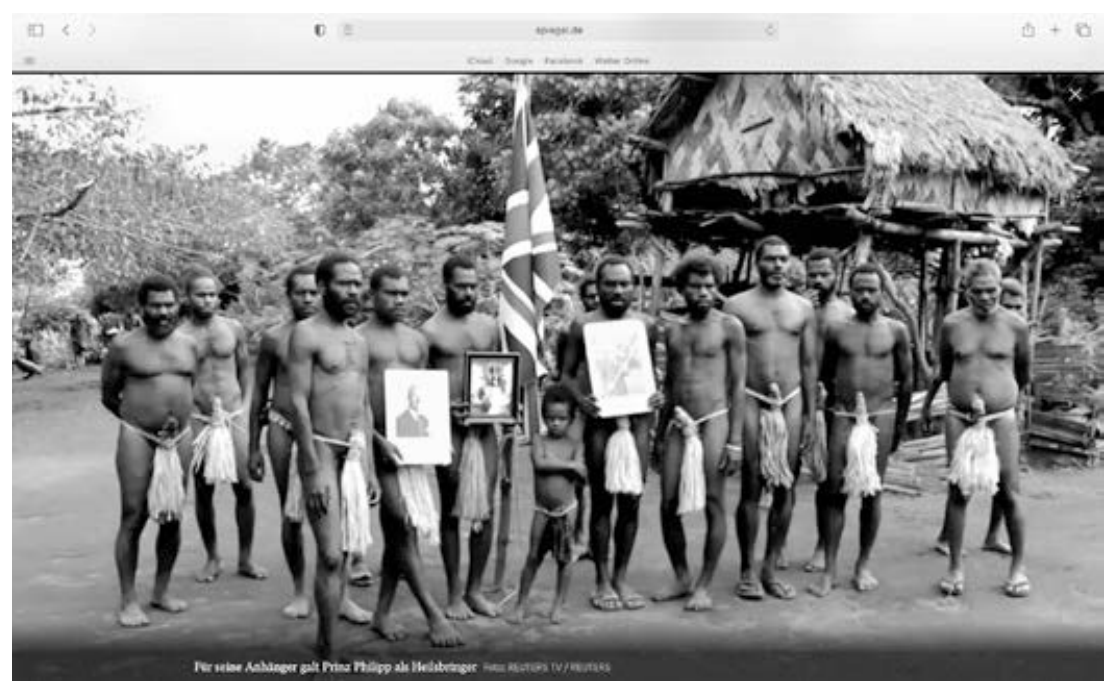

Zeitvorstellungen aufeinandertreffen: die Dauer der Kolonialisierung der Insel, die 1774 mit der Landung von James Cook begann und I980 mit der Unabhängigkeit des Inselstaates Vanuatu ein offizielles Ende fand, und die mythische Zeit(-losigkeit) der Nähe oder Ferne, An- oder Abwesenheit der Ahn_innen. ${ }^{5}$

Einer unverhohlen ethnozentrischen Deutung der Fotografien vom April 202 I sind Journalist_innen des globalen Nordens gefolgt. Denn für sie kam als Erklärung dieser ostentativen Geste nur eines in Betracht: die Heldenbzw. quasi-göttliche Verehrung, die die Schwarzen Männer von Tanna dem weißen Prinzen entgegengebracht haben sollen, weil er ihnen seit seinem ersten Besuch des Archipels im Jahr I 974 nicht einfach als Repräsentant der britischen Krone, sondern als göttlicher Ahne und Wiedergänger des legendären John Frum erschienen sei, der westlichen Quellen zufolge die mythischen <Cargo-Kulte> in den I 940er Jahren wiederbelebt hatte. ${ }^{6}$ Demnach basieren diese Kulte auf der Vorstellung verschiedener indigener Gruppen, dass jeder Ankömmling auf ihrem Land mit der fernen Geisterwelt der Ahn_innen in Verbindung stehe und über Macht, besondere Güter sowie geheimes Wissen verfüge. Mit deren Ankunft werde zugleich die Hoffnung auf ein neues Zeitalter verbunden, das jedoch erst nach einer vollständigen Entledigung von jeglichem Besitz eintrete.

Wie der Anthropologe Lamont Lindstrom betont hat, wurden als <Cargo〉 nicht nur westliche Güter selbst bezeichnet, sondern auch das Wissen, sie herzustellen oder für eigene Zwecke zu verwenden. Im Besitz solch machtvoller Dinge zu sein, versprach demnach Prestige. Lindstrom hat auch darauf hingewiesen, dass <Cargo-Kulte> bis heute ein partikulares Wahrheits- und Machtregime der indigenen Bevölkerung darstellen, das darauf abzielt, sich dem $\mathrm{Zu}$ griff des $\left\langle\right.$ Westens $^{7}{ }^{7}$ zu entziehen - und umgekehrt dem $\langle$ Westen $>$ das Wissen um rituelle Praktiken dazu dient, die indigene Bevölkerung zu beherrschen:
Abb. 1 Spiegel Online, 13.4.2021, Screenshot

5 Zur Kritik an der anthropologischen Konstruktion der vermeintlichen Zeitlosigkeit indigener sprimitiver) Kulturen siehe Johannes Fabian: Time and the Other. How Anthropology Makes Its Object, New York 1983 . Die durch die Fotografien vom April 2021 bezeugte Anteilnahme der Menschen auf Tanna widerspricht dabei direkt dem westlichen Vorurteil der Zeitlosigkeit indigener Gruppen.

6 Peter Worsleys Studie The Trumpet Shall Sound. A Study of "Cargo" Cults in Melanesia (London 1957) versammelt Berichte westlicher Reisender, Missionar_innen und Kolonialbeamter. Die Bezeichnung scargo cults taucht zuerst 1945 in westlichen Zeitungsartikeln auf und steht im Zusammenhang mit dem wachsenden militärischen und ökonomischen Einfluss der USA in der Region. Siehe dazu Lamont Lindstrom: Cargo Cult. Strange Stories of Desire from Melanesia and Beyond, Honolulu 1993, 13-36.

$7 \mathrm{Vgl}$. Stuart Hall: The West and the Rest: Discourse and Power, in: ders., Bram Grieben (Hg.): Formations of Modernity, Cambridge 1992, 275-320. 
8 Lamont Lindstrom: Knowledge of Cargo, Knowledge of Cult: Truth and Power on Tanna, Vanuatu, in: Garry W. Trompf (Hg.): Cargo Cults and Millenarian Movements. Transoceanic Comparisons of New Religious Movements, Berlin 2012, 239-261, hier 241. Lindstrom weist mit Roy Wagner darauf hin, dass die Bezeichnung ‘Kult` bereits eine westliche Zuschreibung für alltägliche rituelle Praktiken indigener Gruppen ist (ebd., 254).

9 Roy Wagner: The Invention of Culture, Chicago, London 1981, 31. Ich danke Jason Papadimas besonders für diesen Hinweis.

10 Ebd., 256. Lindstrom bezeichnet 'Cargo-Kulte) als westlichen Diskurseffekt und 'Cargoismus' als Textgenre der Anthropologie.
«Melanesians attempt to master Europeans by knowing their Cargo. Europeans dominate Melanesians by knowing their cults. $>^{8}$ Der Anthropologe Roy Wagner hat diese reziproke Bezogenheit beider Perspektiven eindrücklich beschrieben:

The words are to some extent <mirror images> of each other, in the sense that we look at the natives' cargo, their techniques and artifacts, and call it <culture, > whereas they look at our culture and call it <cargo.> These are analogic usages, and they betray as much about the interpreters themselves as about the thing interpreted. <Cargo> is practically a parody, a reduction of Western notions like profit, wagelabor, and production for its own sake to the terms of tribal society. ${ }^{9}$

<Cargo-Kulte〉 wurden von der britisch-französischen Kolonialmacht genau deshalb verboten, weil sie die exzessive Zerstörung westlicher Güter als Voraussetzung für das Verschwinden dieser Mächte propagierten und damit auf ihre Weise einen Exorzismus der <Geister des Kapitals〉 betrieben. Sie müssen als eine Reaktion indigener Gruppen auf den kapitalistischen Exzess hinsichtlich des Besitzes von Ressourcen, Menschen und Dingen verstanden werden, der im Kolonialismus und seinen umfassenden Praktiken der Ausbeutung und Unterdrückung anderer Kulturen offensichtlich wird. Gleichzeitig üben diese alles andere als <primitiven> Praktiken bis heute eine anhaltende Faszination auf Menschen des globalen Nordens aus - nicht nur als Projektion eigener Ängste und Wünsche oder mit dem Ziel, andere Menschen zu beherrschen, sondern um Verhalten und Wertvorstellungen innerhalb der eigenen Gesellschaft zu kritisieren. ${ }^{10}$ Sogenannte <Cargo-Kulte> stellen daher immer auch unverhoffte Begegnungen und Erfahrungen mit der eigenen westlichen Kultur dar.

Vor diesem machtpolitischen Hintergrund stellen sich mir folgende Fragen: Handelt es sich bei den Bildern der trauernden Männer von Tanna tatsächlich um nichts anderes als einen Beweis für das Aufleben eines von früheren Kolonialmächten verbotenen Kults oder vielmehr einer religiösen Praktik, die deren Macht gefährdete? Sind sie nur deshalb von Belang, weil sie Fotografien wiedergeben und einen Gestorbenen noch einmal mortifizieren? Ist es möglich, diesen Fotografien auf andere Weise zu begegnen und nicht nur die aktuellen Umstände ihres Entstehens zu beleuchten, sondern gleichfalls die unsichtbaren Handlungen und widerstreitenden Zeiten, in die diese Bilder gleichermaßen verstrickt sind, ein Stück weit zu entfalten? Welche anderen Geschichten und Geschichten von anderen geben die Aufnahmen preis, wenn man sie in die koloniale Geschichte anthropologischer und ethnografischer Aufnahmen einzuordnen versucht, die hegemoniale Blick- und Eigentumsverhältnisse im globalen Süden begründet haben? Und nicht zuletzt: Was besticht mich als westliche Betrachterin an diesen Fotografien?

Die Aufnahmen vom April 202 I sind für mich zunächst bemerkenswert, weil sie die An- und Enteignung westlicher Bildproduktionen durch jene zum Ausdruck bringen, die lange Zeit fast nur als Objekte eines wissenschaftlichkolonialisierenden oder kommerziell-exotisierenden Blicks vorgekommen sind. 
Die indigenen Bevölkerungsgruppen des Pazifiks sind wie die Afrikas, Asiens und der beiden Amerikas seit Mitte des I9. Jahrhunderts in beispielloser Weise Objekte eines <kolonialen Blicks〉 geworden und immer wieder mit Kameras konfrontiert worden." Wie David Bate betont, sind die «Geschichte des Kolonialismus und die Geschichte der Fotografie [...] in der industriellen und imperialistischen Expansion Europas im I9. Jahrhundert eng miteinander verflochten». ${ }^{2}$ Auch wenn die Männer von Tanna im April 202 I nicht selbst die Kamera führten, sondern sich wiederum ablichten ließen, so bleiben doch der langjährige Besitz repräsentativer Fotografien ebenso wie die Vertrautheit mit fotografischen Aufnahmeprozeduren das über das fotografierte Ereignis hinausgehende Ereignis der Fotografie, das zu einer genaueren Betrachtung der Fotografien und der mit ihnen vollzogenen Handlungen auffordert.

Im Folgenden möchte ich daher Ariella Azoulays Unterscheidung zwischen dem fotografierten Ereignis und dem Ereignis der Fotografie aufgreifen, um meinen Blick auf das Handlungsgefüge zwischen dem Repräsentanten einer ehemaligen Kolonialmacht und der Bevölkerung von Vanuatu zu richten, das durch Fotografien etabliert und vermittelt wird. Azoulay fasst als fotografiertes Ereignis das, was Fotografien wie und durch wen (nicht) zu sehen geben, und bezeichnet die vielfachen und andauernden, erwarteten wie zufälligen Begegnungen mit Fotografien, ihrer Verbreitung, Zirkulation und Interpretation durch andere an anderen Orten zu anderen Zeiten als Ereignis der Fotografie. ${ }^{13}$ Ich verstehe die durch Nachrichtenkanäle und Social-Media-Plattformen verbreiteten Bilder-Bilder als über verschiedene Orte, Zeiten und Handelnde verteiltes Ereignis der Fotografie, zu dem ich mich in Beziehung setze. Mich interessiert dabei besonders, welche Rolle Fotografien überhaupt und die royalen Porträts und Familienfotos im Leben der Menschen von Tanna gespielt haben könnten - Bilder, die sie offenbar über Jahrzehnte aufbewahrt haben. Diese Praktik fällt allein schon deshalb auf, weil viele Dinge im feuchtheißen Klima der Insel nicht konserviert werden können. ${ }^{14}$ Fotografien sind fragil und lösen sich mit der Zeit auf - auch ihre grundsätzlich begrenzte zeitliche Dauer ist Teil der Geschichte dieser Fotografien. Sie beginnt mit einem Tausch von Gaben.

\section{(Bilder-)Gaben}

Auf einer von der Nachrichtenagentur Reuters verbreiteten Fotografie sieht man einen älteren indigenen Mann vor seiner Hütte. Seinen Namen erfahren wir nicht. Er blickt direkt in die Kamera und hält eine großformatige Porträtfotografie von Philip mit beiden Händen wie ein Schild vor seinen nackten Oberkörper (Abb. 2). Der darauf Abgebildete, förmlich im Anzug gekleidet, befindet sich in einem englischen Park und hält mit einer ähnlichen Geste einen auffällig verzierten hölzernen Schläger in Händen. Dabei springt unmittelbar der Kontrast zwischen der Nacktheit des einen und der westlichen Kleidung des anderen ins Auge. Meinen Blick irritiert jedoch besonders die Äquivalenz
11 Zur Ambivalenz des kolonialen Blicks zwischen Schaulust und Identifikation siehe David Bate: Fotografie und der koloniale Blick, in: Herta Wolf (Hg.): Diskurse der Fotografie. Fotokritik am Ende des fotografischen Zeitalters, Frankfurt/M. $2003,115^{-132 .}$

12 Ebd., 115.

13 Siehe Ariella Azoulay: Civil Imagination. A Political Ontology of Photography, London, New York 2012. 14 Siehe Lindstrom: Knowledge of Cargo, Knowledge of Cult, 247: "Storage and conservation are rare in the Melanesian environment. [...] [T]ools and clothing must be constantly renewed.» 


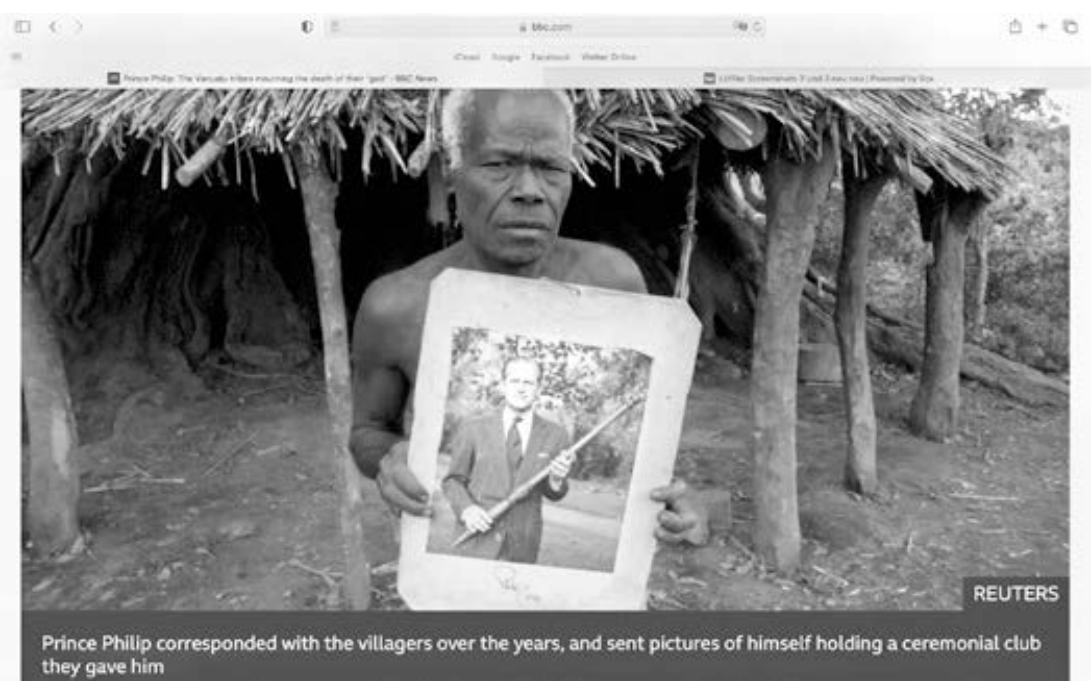

Abb. 2 BBC News, 12.4 .2021 , Screenshot

15 Huffman ist Kurator am Australischen Museum in Sydney und war von 1976 bis 1989 Kurator am Vanuatu Cultural Centre.

16 Vgl. Wong: Prince Philip.

17 Siehe dazu Sara Ahmed: Strange Encounters. Embodied Others in PostColoniality, London, New York 2000. der Zeigegesten: Sie besticht als demonstrativer Akt eines gestischen Dialogs, der zwei Orte, Zeiten, Personen und Kulturen zueinander über den vorgezeigten Gegenstand in eine bildliche Relation setzt. Wie kommt es zum fotografierten Ereignis dieser (unabsichtlichen) Äquivalenz von Fotografie und hölzernem Schläger? Was verbindet sie? Und: Wie ist die offenbar signierte Fotografie des britischen Royal nach Vanuatu gelangt?

Die $B B C$-News-Journalistin Tessa Wong hat dazu anlässlich von Prinz Philips Tod den Anthropologen Kirk Huffman befragt, der seit Jahrzehnten indigene Gruppen auf Vanuatu besucht und die Sache aufzuklären weiß: Die signierte Fotografie ist eine Gegengabe. ${ }^{15}$ Als Philip 1978 den Inselstaat zum zweiten Mal besuchte, wurde der royale Besucher auch von Männern aus Tanna begrüßt, die seinem Schiff in ihren Kanus entgegenfuhren. Persönlich getroffen hat er diese Männer nicht. An seiner statt wurde ein Bevollmächtigter des Vereinigten Königreichs mit einer Auswahl von Fotografien des Prinzen zu ihnen geschickt. Diese Fotografien wurden von einem lokalen Chief mit einer Gegengabe beantwortet: Dem Gesandten wurde ein nal-nal genannter Schläger überreicht, der bei Zeremonien sowie der Schweinejagd eingesetzt wird. Das Foto, das den Prinzen mit seinem nal-nal zeigt, ist also der Beleg, dass er das Geschenk tatsächlich erhalten hat. So hat es Huffman der $B B C$-Journalistin berichtet. Er kennt diese Geschichte, weil er nach eigener Aussage bei der Übergabe der verzierten Waffe dabei war. ${ }^{16}$

Der Tausch von Gaben zwischen verschiedenen Gruppen ist die Ursprungsszene des Kulturkontakts, die notorisch wiederholt wird: das erste Aufeinandertreffen zweier einander fremder Kulturen, die am Beginn des Kolonialismus und der kolonialistischen Konstruktion eines vermeintlich <primitiven> Anderen steht. ${ }^{17}$ Dieser Erstkontakt ist von unterschiedlichen Sprachen, Kulturtechniken, Waffen und Ressourcen geprägt, die ein Machtgefälle erzeugen. Im Fall von 
Prinz Philip ist auch die zweite Begegnung mit den Leuten von Vanuatu I978 asymmetrisch. Die Inselgruppe ist zu diesem Zeitpunkt britisch-französisches Herrschaftsgebiet, deren Verwaltung die ökonomischen Interessen beider Länder sichert, ohne der indigenen Bevölkerung staatsbürgerliche Rechte zugestehen zu müssen. Philip bleibt in Port Vila auf der Insel Efate und schickt einen Vertreter nach Tanna - mit Porträtfotografien im Gepäck. Dort kommt es in seiner Abwesenheit zum Gabentausch. Der nal-nal wechselt in den Besitz des abwesenden Prinzen. Im Gegenzug erhalten die Männer von Yaohnanen und Yakel jene Fotografie des Prinzen, der die geschenkte Waffe in Händen hält - signiert als Geste der Authentifizierung und Ausdruck kultureller Distinktion.

Diese von Huffman vorgetragene Erzählung klärt jedoch nicht, welchen Stellenwert die Fotografien von Prinz Philip und seiner Familie und Fotografien überhaupt für die Menschen von Tanna haben. Was demonstrieren die im April 202 I aufgenommenen Bilder, wenn nicht Helden- oder Götterverehrung, wie die Berichterstattung im globalen Norden suggeriert? Andere Gebrauchsweisen dieser Aufnahmen sind denkbar, wenn man das Handlungsgefüge zwischen dem Repräsentanten einer ehemaligen Kolonialmacht und den ni-Vanuatu, wie sich die Bevölkerung des Inselstaates selbst nennt, genauer in den Blick nimmt. Dabei gilt es auch, den Bilderkult der eigenen westlichen Kultur und seine Projektion auf die vermeintlich <primitiven> Anderen ins Visier zu nehmen und als gegenläufige Idolatrie zu begreifen. ${ }^{18}$ Statt Helden- oder Heiligenverehrung kommen andere Erklärungen ins Spiel, die nicht weniger plausibel sind. Was wird beim Gabentausch verhandelt, wenn nicht Prestige als Ausdruck kultureller Wertvorstellungen? Geht es bei der Tauschkette Fotografie gegen nal-nal gegen Fotografie mit nal-nal nicht eher um die Möglichkeit einer gegenseitigen Aushandlung von Prestige und Macht, wie Lamont Lindstrom am Beispiel der <Cargo-Kulte> gezeigt hat? Oder mehr noch: um die Bedingung der Möglichkeit einer reziproken Anthropologie oder «reverse anthropology» in Roy Wagners Sinn? ${ }^{19}$ Und würden die Porträtfotografien dann nicht als immutable mobiles im Sinne Bruno Latours ${ }^{20}$ den Zweck erfüllen, diese Gleichwertigkeit zu bezeugen? So wird überhaupt erst verständlich, warum die Männer in Yaohnanen und Yakel die Fotografien des Prinzen in die Kamera halten.

Die Fotografien bezeugen so gesehen weniger Ereignisse als den Vollzug von Handlungen, den Eintritt in eine zirkuläre Ökonomie des Tauschens, die vor allem die Aushandlung der gegenseitigen Beziehung zum Gegenstand hat. Der Wert der Fotografien von Prinz Philip für die ni-Vanuatu besteht daher aus meiner Sicht im Verdeutlichen von Interessen bzw. Wertvorstellungen und nicht allein in der Person, die sie abbilden. Denn die Aufnahmen in ihren Hütten aufzubewahren muss gleichwohl nicht bedeuten, sie aufzuhängen und ständig im Blick zu haben, um das fotografierte Ereignis dadurch zu einem Ereignis der Fotografie werden zu lassen. ${ }^{21}$ Sie werden im Gegenteil nur zu besonderen Anlässen hervorgeholt und nach dem Tod der porträtierten Person verhüllt..22 Ich schlage daher vor, die Fotografien weniger als Schau-Objekte, denn als arkane
18 Als Beispiel für den westlichen Bilderkult ist gerade die populäre Idolatrie britischer Royals nicht nur in Großbritannien schlagend. So wurde im April 2021 am Piccadilly Circus in London ein haushohes Werbebanner mit einem Porträt des Verstorbenen angebracht.

19 Wagner: The Invention of Culture, 30.

20 Siehe Bruno Latour: Drawing Things Together, in: Michael Lynch, Steve Woolgar (Hg.): Representation in Scientific Practice, Cambridge (MA), London 1990, 19-68. Für Latour sind Bilder, Diagramme und Karten ebenso unveränderliche wie mobile Mittel des Wissens und damit auch der Ausübung von Herrschaft.

21 Im Kontrast zu diesen Fotografien im Besitz der Leute von Tanna waren Porträts des britischen Königspaars während und nach der Kolonialzeit, als sie das britische Empire auf dem von ihnen «New Hebrides' genannten Inselstaat repräsentierten, in öffentlichen Gebäuden verbreitet und keineswegs ungewöhnlich.

22 Roger Boulay beschreibt diese Praktiken des Nicht-Zeigens bei den Kanak auf Neukaledonien, siehe ders.: po hânu - In seinem Schatten aufrecht gehen. Erläuterungen zur Wahrnehmung der Fotografie bei den Kanak, in: Museum für Völkerkunde Basel (Hg.): Portraits kanak-paroles kanak. Historische Fotografien von Fritz Sarasin. Zeitgenössische Texte aus Neukaledonien, Basel 1996, 76-85, hier 81 . 


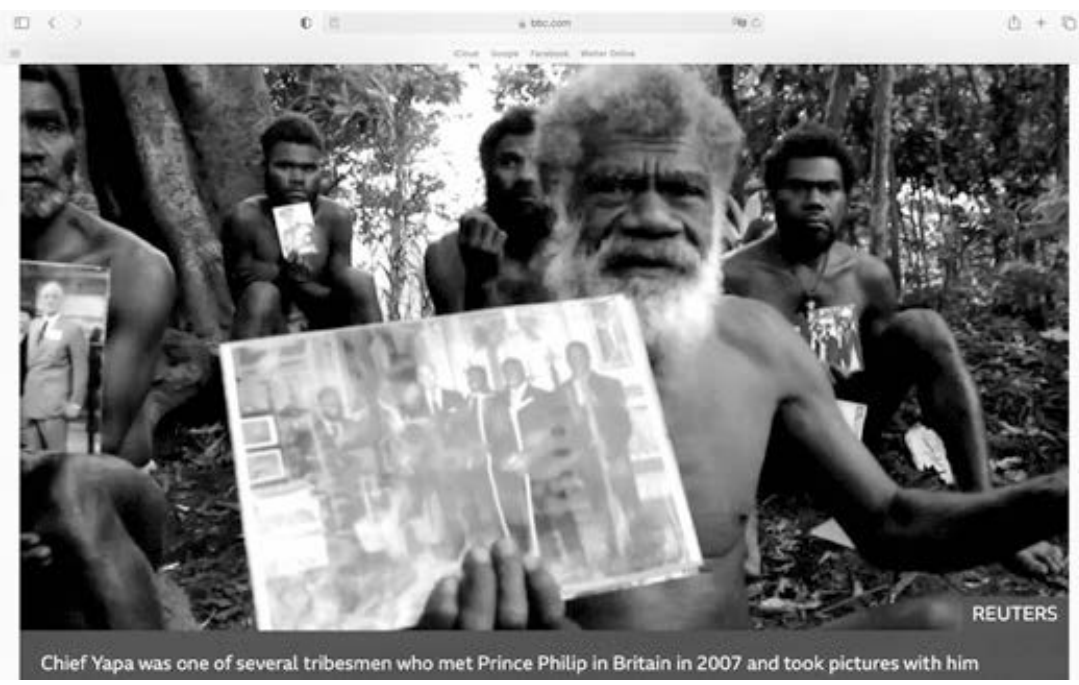

Abb. 3 BBC News, 12.4 .2021 , Screenshot

23 Lindstrom weist darauf hin, dass auch Bücher und eine mit Fotografien versehene Broschüre der US-Armee als Cargo fungierten, ders.: Knowledge of Cargo, Knowledge of Cult, 250.

24 Meet the Natives, Regie: Gavin Searle, BBC Channel 4, GB 2007.

25 Siehe zum Voyeurismus des globalen Nordens Alison Griffiths: Wondrous Difference. Cinema, Anthropology, and Turn-of-the-Century Visual Culture, New York 2002.

$26 \mathrm{Zu}$ solchen Formen mimetischer Alterität siehe Michael Taussig: Mimesis and Alterity. A Particular History of the Senses, London 1993.
Objekte, also selbst als <Cargo〉 zu betrachten, dessen Besitz Wissen und damit auch Macht innerhalb der eigenen Gruppe und gegenüber (post-)kolonialen Autoritäten verspricht. ${ }^{23}$ Denn mit dem Besitz von Fotografien bemächtigen sich die Leute von Tanna zugleich einer westlichen Bildpraktik.

Diese Erklärung erhält Plausibilität nicht zuletzt durch den Umstand, dass fünf Abgesandte der Insel 2007 nach Großbritannien gereist und dort Philip schließlich doch begegnet sind. Auch von dieser Begegnung gibt es Fotografien, die ebenfalls im April 202 I von denjenigen in die Kameras gehalten wurden, die wie Chief Yapa damals dabei waren (Abb. 3). Anlässlich dieses Besuchs produzierte die $B B C$ eine dreiteilige Fernsehdokumentation. Ihr Titel, Meet the Natives, ${ }^{24}$ erinnert zwar an koloniale Völkerschauen des I9. Jahrhunderts, ${ }^{25} \mathrm{dreht}$ den Spieß aber um: Die «Natives» sind in diesem Fall Brit_innen - mit einer Videokamera gefilmt bei ihren Gebräuchen von den Gesandten aus Tanna. Die Dokumentation zeigt, wie Letztere sich nicht nur im Sinn der <Cargo >-Ideologie eine westliche Bildtechnologie aneignen und mit ihrer Hilfe eine reziproke Anthropologie betreiben, sondern auch wie sie Tourist_innen auf Bilderfang imitieren. ${ }^{26}$ Es hat ganz den Anschein, als ob der Besuch Philips nur den naheliegenden Anlass für eine touristische Reise und ihre obligatorischen Bildpraktiken abgibt. Dafür spricht auch, dass die Dokumentation eine Fortsetzung nach dem gleichen Muster erfahren hat, dieses Mal mit dem Reiseziel USA.

\section{Koloniale (Parallel-)Geschichten der Fotografie}

Auch die Fotografie hat ihre kolonialen Mythen. Die Anthropologie hat sich als durch und durch westliche Wissenschaft in der zweiten Hälfte des I9. Jahrhunderts schon früh der Fotografie bedient, um ethnisch und kulturell Andere nach westlichen Maßstäben zu ordnen und sie damit zu Objekten 
eines rassifizierenden Blicks zu machen. ${ }^{27}$ Carl Dammanns I 873 erschienenes Anthropologisch-Ethnologisches Album in Photographien ${ }^{28}$ stellt eine der ersten Bildergalerien ethnisch differenzierter Menschentypen dar. Die rund 600 Aufnahmen stammen zumeist von europäischen Fotografierenden, die sie an Reisende, Handel und Wissenschaft Treibende aus dem globalen Norden verkauften. Auch die Berliner Gesellschaft für Anthropologie, Ethnologie und Urgeschichte kaufte solche kommerziellen Aufnahmen im carte-de-visite-Format an und ließ sie vom Hamburger Fotografen Carl Dammann reproduzieren und verbreiten. ${ }^{29}$ Wie Elizabeth Edwards betont, bestand die Rolle der Fotografien darin, «virtuelle Zeugen wissenschaftlicher Beobachtungen und der Herstellung wissenschaftlicher Tatsachen wie z. B. Taxonomien zu schaffen».30 Sie hat zugleich die rassifizierenden Klassifizierungen und Typisierungen der sogenannten <Wissenschaften vom Menschen> und deren Verstrickung in koloniale Machtausübung und Besitzergreifung aufgedeckt.

Fotografien spielten auch eine wichtige Rolle bei der Etablierung der ethnografischen Feldforschung Anfang des 20. Jahrhunderts. Wissenschaftler_innen vor allem der Kolonialmächte Großbritannien, Frankreich und Deutschland reisten nun selbst in die Pazifikregion und nahmen vor Ort in großem Umfang Daten über indigene Bevölkerungen auf, rückten mit Messstab, Foto- und Filmkamera anderen Menschen auf den Leib und bemächtigten sich ihrer als Versuchsobjekte. Mit gleicher Gründlichkeit und Vehemenz bemächtigten sie sich ritueller Artefakte und Gebrauchsgegenstände, um damit in Europa und Nordamerika bedeutende anthropologische und ethnografische Sammlungen anzulegen und Museen zu bestücken. ${ }^{31}$ Diese Praktiken der Extraktion als Sammeln zu bezeichnen, wie das heute noch immer geschieht, ist ein Euphemismus, der die gängigen kolonialen Entnahmepraktiken, darunter Zwang und Raub, verschleiert, die wissenschaftliche Expeditionen bis weit ins 20. Jahrhundert hinein angewendet haben. ${ }^{32}$

Fotografien und später auch Filmaufnahmen dienten dabei nicht nur als (glaubhafte) Belege für propagierte Forschungsergebnisse, sondern auch für Ereignisse wie z.B. die Aufführung bestimmter Riten oder Tänze. Und wenn es gelang, besonders seltene oder geheime Praktiken vor Ort im Bild festzuhalten, wurden sie geradezu zu begehrten Bildtrophäen, die unter Forschenden zirkulierten und vielfach publiziert wurden. Der britische Zoologe und Anthropologe Alfred Cord Haddon, der I 898 die groß angelegte, von der britischen Anthropologischen Gesellschaft finanzierte Torres-Straits-Expedition leitete, nahm zwischen I908 und 1935 mehrere hundert Fotografien im Archipel auf. ${ }^{33}$ Haddon benutzte seine Fotografien nicht nur als visuelle Feldnotizen, sondern auch als Tauschobjekte und schenkte sie indigenen Personen, die sich freiwillig ablichten ließen, als <Belohnung $>{ }^{34}$ Auch auf Vanuatu wurde eifrig fotografiert. Der britische Anthropologiestudent John Layard, der I9I4/I 5 mehrere Monate auf der Insel Atchin mit intensiver Feldforschung verbrachte und unter anderem $\mathrm{zu}<$ Cargo-Kulten $>$ forschte, fertigte über 400 Aufnahme auf Glas an. ${ }^{35}$
27 Siehe Elizabeth Edwards (Hg.): Anthropology and Photography 1860-1920, New Haven, London 1992.

28 Siehe Carl Dammann: Anthropologisch-Ethnologisches Album in Photographien, Berlin 1873.

29 Siehe Elizabeth Edwards: Shifting Representation. The Making of the Ethnographic in 19th Century Photography, in: HansPeter Bayerdörfer u.a. (Hg.): Bilder des Fremden. Mediale Inszenierung von Alterität im 19. Jahrhundert, Berlin 2007, 41-62, hier $43 \mathrm{f}$.

30 Elizabeth Edwards: Andere ordnen. Fotografie, Anthropologien und Taxonomien, in: Herta Wolf (Hg.): Diskurse der Fotografie. Fotokritik am Ende des fotografischen Zeitalters, Frankfurt/M. 2003 , 335-355, hier 337.

31 Siehe dazu Chris Gosden, Chantal Knowles (Hg.): Collecting Colonialism. Material Culture and Colonial Change, Oxford u.a. 2001; aktuell Götz Aly: Das Prachtboot. Wie Deutsche die Kunstschätze der Südsee raubten, Frankfurt/M. 2021.

32 Fritz Kramer und Hans Fischer haben schon vor 40 Jahren explizit auf die koloniale Verstrickung deutscher Ethnologen wie Adolf Bastian und wissenschaftlicher Expeditionen wie der Hamburger Südsee-Expedition (1908-1910) hingewiesen, Fritz Kramer: Verkehrte Welten. Zur imaginären Ethnographie des 19. Jahrhunderts, Frankfurt / M. 1979, 74-81; Hans Fischer: Die Hamburger Südsee-Expedition. Über Ethnographie und Kolonialismus, Frankfurt/M. 1981.

33 Allein das Museum für Archäologie und Anthropologie der Universität Cambridge beherbergt 589 Abzüge, siehe Geismar: Malakula, 521.

34 Siehe Griffiths: Wondrous Difference, 145. Wie Haddon berichtete, waren Fotografien sehr begehrte Geschenke.

35 Siehe Geismar: Malakula, 532. 
Auf diese Weise ist an westlichen Universitäten und Museen des globalen Nordens ein immenses fotografisches Archiv entstanden, das die Reichweite und Dominanz kolonialer Wissenspraktiken dokumentiert. Der Besitz von Fotografien wie von kulturellen Gütern war ein probates Mittel, durch Wissen zu Anderen gemachte Menschen zu beherrschen. Aber nicht nur Forschende des globalen Nordens fotografierten und filmten, auch Eingewanderte eigneten sich die Technik an, fotografierten die indigene Bevölkerung, verkauften die Aufnahmen gewinnbringend und etablierten so einen weltweiten Bildermarkt. ${ }^{36}$ «Die Nachfrage nach <exotischen〉 Bildern wuchs in Europa und Nordamerika seit <Erfindung〉 der Fotografie immens an und eröffnete ständig neue Märkte», wie Alison Devine Nordström betont, die die Zirkulation von Fotografien von der Pazifikinsel Samoa untersucht hat. ${ }^{37}$ Dabei kursierten vor allem Postkarten zwischen den pazifischen Inseln und dem Rest der Welt, die exotisierte Vorstellungen indigener Menschen und Kulturen nährten.

Die Anthropologin Haidi Geismar untersucht die Migrationsbewegungen von Fotografien zwischen Vanuatu und dem Rest der Welt, die von Forschern wie Haddon oder Layard im Rahmen ethnografischer Feldforschung aufgenommen wurden und ihren Weg in wissenschaftliche Publikationen, Museen und Archive weltweit fanden. Sie begleitet vor allem Projekte, bei denen Abzüge dieser Fotografien aus Archivdepots wie der Haddon Collection an der Universität von Cambridge wieder nach Vanuatu, den Ort ihres Entstehens, zurückgebracht werden, um sie erneut in lokale kulturelle Praktiken zu integrieren und gleichzeitig zu aktivieren. Denn aus diesen Bildern kann für Geismar historisches Wissen über Artefakte und Rituale gewonnen werden, die in der Kolonialzeit unterdrückt wurden:

$[\mathrm{P}]$ hotographs are used explicitly as conduits into social action, they are re-embedded into practices, becoming, in the present, part of what they once seemed merely to represent from the past. Images are used in preparations for ritual dances, matmaking, painting, story-telling, and so on. ${ }^{38}$

Geismar geht es wie auch Azoulay darum, fotografische Praktiken als dynamisches Gefüge zwischen Fotografierenden, Fotografierten_m und Fotografien

36 Siehe dazu die Studie von Anne Maxwell: Colonial Photography \& Exhibitions. Representations of the (Native) and the Making of European Identities, London, New York 1999.

37 Alison Devine Nordström: Populäre Fotografie aus Samoa in der westlichen Welt - Herstellung, Verbreitung und Gebrauch, in: Jutta Beate Engelhard, Peter Mesenhöller (Hg.): Bilder aus dem Paradies. Koloniale Fotografie aus Samoa 1875-1925, Marburg 1995 , 13-39, hier 13 .

38 Geismar: Malakula, 526.

39 Siehe ebd., $55^{8}$. zu beschreiben, das explizit Praktiken der Bildmigration umfasst. Dieser Bilderverkehr zwischen Vanuatu und Großbritannien weist Fotografien explizit die Rolle eines Mediators zu, die über ihre Funktion als immutable mobiles hinausweist. Denn sie legen nicht einfach Zeugnis über Handwerkstechniken oder rituelle Praktiken ab, sondern übermitteln Botschaften aus der mythischen Zeit und der kolonialen Vergangenheit, derer sich die ni-Vanuatu wieder bemächtigen wollen - und die im besten Fall eine Ethnografie der eigenen Kultur, ihrer Vergangenheit und ihres Wandels erlaubt. Sich diese in die Geschichte des Kolonialismus verstrickten Fotografien als historische Artefakte der eigenen Kultur anzueignen, stellt für Geismar alles andere als einen Akt der Idolatrie dar - es macht sie vielmehr zu machtvollen <Agenten der Geschichte> ${ }^{39}$ Vor 
diesem Hintergrund sind nicht nur irritierende Begegnungen mit Fotografien und fotografischen Praktiken, sondern auch überraschende Wiederbegegnungen mit den eigenen Vorfahren und lokalen Traditionen möglich. Jedes fotografierte Ereignis wird erst so zu einem Ereignis der Fotografie in Azoulays Sinn. Fotografien sind dabei Teil von transkulturellen Verhandlungen verschiedener kultureller Werte und Interessen. Das erfordert jedoch zugleich, sie selbst in gewisser Weise zu entmachten.

\section{Abweisung eines Idols}

Dass der Umgang mit westlichen Fotografien auch widerständige Praktiken hervorbringt, belegt der Film Tanna (Australien/Vanuatu 2015), der auf der gleichnamigen, zu Vanuatu gehörenden Insel von Martin Butler und Bentley Dean in Kollaboration mit der indigenen Bevölkerung nach einer wahren Begebenheit gedreht wurde. Das Drama um eine verbotene Verbindung zweier Liebender wurde für einen Oscar nominiert und gewann bei den 72. Filmfestspielen von Venedig den Publikumspreis. Die ni-Vanuatu haben jedoch stets darauf bestanden, dass der Film ibr Leben zwischen Tradition und Transformation zeige und daher ganz und gar ihr eigener sei. Und es ist ein Film, in dem die Wünsche und Handlungen junger Frauen und Mädchen eine wichtige Rolle spielen, die sich nicht ohne Widerstand tradierten Geboten ihrer Gemeinschaft oder dem 〈Vorbild〉 westlicher Kultur unterwerfen wollen, auch wenn das zu ernsthaften kriegerischen Konflikten führt.

In diesem Konflikt spielen auch Fotografien des britischen Königpaars eine wichtige Rolle. Es handelt sich um Familienbilder aus einer alten englischsprachigen Zeitschrift. Der jungen Wawa (Marie Wawa) werden diese Bilder von ihrem Großvater (Albi Nanga), dem lokalen Schamanen der Siedlung Yakel, gezeigt, um sie zur Ehe mit einem Angehörigen einer feindlichen Gruppe zu überreden - mit der Begründung, auch das britische Paar wäre eine arrangierte Ehe eingegangen. Dem alten Mann fällt die Aufgabe zu, Wawa die Notwendigkeit exogamer Eheschließungen für das friedliche Zusammenleben mit Anderen und das Fortbestehen der eigenen Gruppe zu erklären. Dieses mündlich vermittelte Wissen um Traditionen, die in der lokalen Sprache, dem Bislama, kastom genannt werden, ${ }^{40}$ unterstreicht der Schamane durch die <Cargo>-Macht der royalen Fotografien. Nachdruck verleiht er seiner Deutung der glücklichen Verbindung von Philip und Elizabeth durch eine weitere Fotografie, die ihn 2007 als Abgesandten von Tanna zusammen mit dem britischen Prinzen zeigt (Abb. 4). Es ist die gleiche Fotografie, die Chief Yapa im April $202 \mathrm{I}$ in die Kamera hielt (siehe Abb. 3).

Die beiden Fotografien in dieser Szene des Films sollen einerseits Autorität vermitteln und andererseits das Ereignis der Begegnung bezeugen. In der filmischen Handlung wird jedoch die Konvention einer arrangierten Partnerschaft, die aus Wawas Sicht ebenso unzeitgemäß wie unattraktiv ist, hinterfragt und
40 Auf der Insel wird Bislama, das nationale Pidgin-Englisch, neben zahlreichen indigenen Sprachen bzw. Dialekten sowie Englisch und Französisch gesprochen, siehe Lamont Lindstrom: Tanna Times. Islanders in the World, Honolulu 2020, 5. 


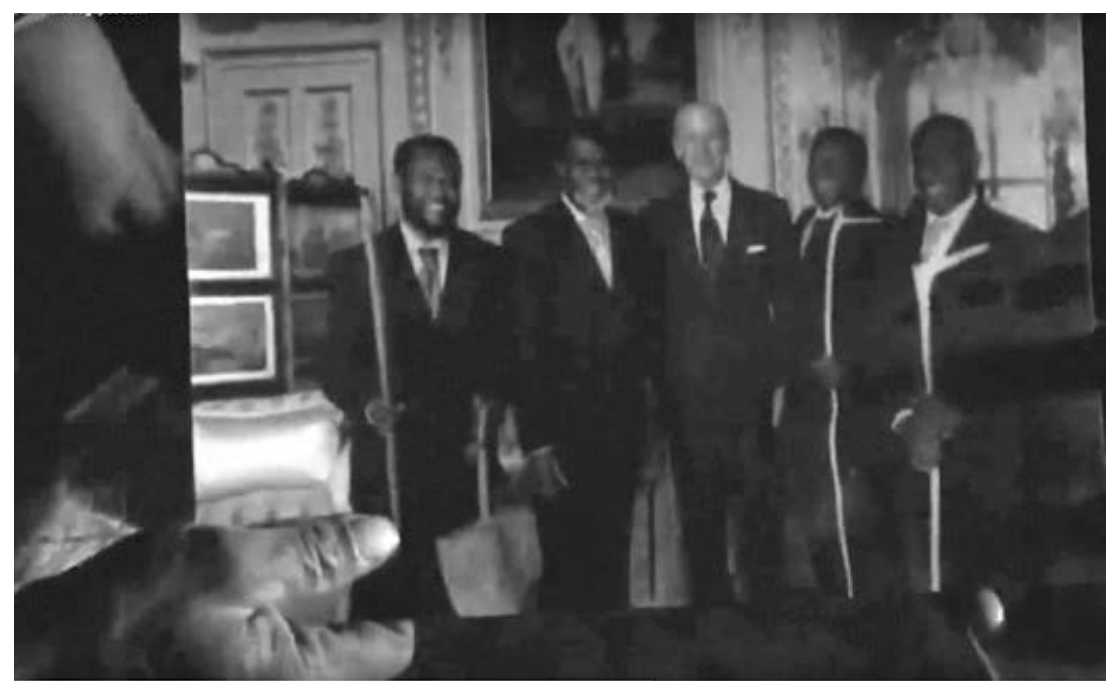

Abb. 4 Albi Nanga weist auf das Foto, das ihn 2007 zusammen mit Prince Philip und anderen ni-Vanuatu zeigt. Filmstill aus: Tanna, Regie: Martin Butler und Bentley Dean, Australien/ Vanuatu, 2015
41 Zu den Reaktionen auf die Vorführung des Films in Port Vila vgl. Lindstrom: Tanna Times, 127. von den jungen Menschen als vermeintliches Vorbild abgelehnt. Denn Wawa hat längst ihre Wahl getroffen und ist durch nichts von ihrer Liebe zu Dain (Mungau Dain) abzubringen - auch nicht durch die royalen Familienfotos oder die Autorität des Schamanen und dessen <Cargo>-Wissen. Der Konflikt zwischen selbstbestimmter Liebe und Unterordnung unter verbindliche Regeln treibt das Paar in den freiwilligen Tod. Aus Trauer um den Verlust zweier für das Überleben der eigenen Gruppe wichtiger Menschenleben kommt es nicht nur zu einer Versöhnung der verfeindeten Clans, sondern auch zu einer Änderung der Heiratsvorschriften: Die romantische Liebe wird als Option in das Regelwerk des kastom aufgenommen. Ganz nebenbei zeigt der Film, dass die Leute von Tanna ihren eigenen Weg suchen. ${ }^{41}$

Die im Film praktizierte Verwendung royaler Fotografien unterscheidet sich insofern von dem ostentativen Gebrauch, den die Männer von Tanna jüngst an den Porträts des verstorbenen Prinzen vollzogen. Während sie vorrangig Porträts des verstorbenen Prinzen öffentlich vorzeigten, um Prestige als kulturellen Wert zu evozieren, werden im Film Fotografien der Royals im nichtöffentlichen Rahmen einer Unterweisung verwendet. Ihre disziplinierende <Cargo>-Macht können sie jedoch nicht mehr ausüben, weil die mit der westlichen Darstellung konventionellen Familienglücks verbundenen Wertvorstellungen von der jüngeren Generation nicht mehr geteilt werden. Hier zeigt sich auch eine wesentliche genderspezifische Asymmetrie: Der Besitz von Fotografien und damit <Cargo> liegt in der Hand von Männern, ist ebenso Chefsache wie der Besuch in Großbritannien. Umso bedeutsamer ist es, dass die junge Frau in der Fiktion des Films die Vorbildfunktion der Royals und die bildmächtige Inszenierung westlicher Werte ablehnt. Ihre Zurückweisung des westlichen Idols ist auch eine Zurückweisung der <Cargo >-Macht der Fotografie, die ich in Anlehnung an Ariella Azoulay als Nicht-Ereignis der Fotografie 
bezeichnen möchte. Im Unterschied dazu weisen die Männer von Tanna, die im April 202 I Fotografien von Philip in die Kamera hielten, den <kolonialen Blick> durch seine Umkehrung ab: Im Spiegel des royalen Porträts erscheint der westliche Mensch - oder besser: Mann - sich selbst in seiner ganzen ungelenken Fremdheit.

\section{Postskriptum}

Am I4. und I 5. April 202 I, nur wenige Tage nach Prinz Philips Tod, veranstaltete das Vanuatu Kaljoral Senta in Port Vila ein zweitägiges Symposium über die Geschichte der nationalen Identität (History Mo Nasonal Identiti). Im überfüllten Malvatumauri Nakamal, dem lokalen Versammlungshaus, wurde die Forderung laut, mehr als 40 Jahre nach der Unabhängigkeit von Vanuatu selbst Archäologie und Anthropologie zu betreiben: «Vanuatu needs more archaeologists and anthropologists», ${ }^{42}$ wie Richard Shing, der Direktor des Kulturzentrums und selbst Archäologe, betonte. Seine Initiative umfasst explizit auch die mehr als I 30 Sprachen, die im Archipel gesprochen werden und von den Kolonialmächten unterdrückt wurden. Zum Studium kultureller Praktiken empfiehlt er wiederum etablierte Techniken und Methoden westlicher Wissenschaft - «to film, record and collect cultural data by utilizing current technology for safekeeping purposes». ${ }^{43}$ Das Wissen, das Fotografien oder Filme bergen, hat scheinbar nichts von seiner Faszination verloren: Koloniale Bildpraktiken werden zum postkolonialen Wiedergänger ihrer selbst - und zum Anlass aktueller Interventionen in diese Archive, durch die Fotografien wiederum zum Ereignis in Azoulays Sinn werden: «Photography is now being rephotographed.» ${ }^{44}$
42 o. A.: Vanuatu needs more archaeologists and anthropologists [...], Post auf Vanuatu Kaljoral Senta (Facebook-Seite), 7·5.2021, facebook. com/VanuatuKaljoralSenta/photos|a.1327216314046345/339674973375 9649/ (7.6.2021).

43 Ebd.

44 Christopher Pinney: The Parallel Histories of Anthropology and Photography, in: Elizabeth Edwards (Hg.): Anthropology and Photography 1860-1920, New Haven, London 1992, 74-95, hier go. Pinneys Worte gelten der kritischen Durchleuchtung kolonialer Archive. 